BULLETIN OF THE

AMERICAN MATHEMATICAL SOCIETY

Volume 81, Number 1, January 1975

\title{
A CONSTRUCTIVE CHARACTERIZATION OF DISCONJUGACY
}

\author{
BY ANTON ZETTL 1
}

Communicated by Fred Brauer, August 2, 1974

An ordinary linear differential operator $L$ defined by

$$
L y=y^{(n)}+a_{n-1}(t) y^{(n-1)}+\cdots+a_{1}(t) y^{\prime}+a_{0}(t) y
$$

is said to be disconjugate on an interval $I$ if every nontrivial solution of

$$
L y=0
$$

has less than $n$ zeros on $I$, multiple zeros being counted according to their multiplicity.

We assume $a_{i} \in C(I)$ for $i=0, \cdots, n-1$. This assumption is made mainly for convenience and can be considerably weakened.

We announce here an algorithm for the construction of disconjugate operators of type (1) for any $n \geqslant 2$ and all intervals $I$. Our construction yields all disconjugate operators of type (1) if the interval $I$ is either open or compact. This construction has the following features: It is iterative or inductive in the sense that the set of $n$th order disconjugate operators is constructed from the set of $(n-1)$ st order ones. (The second order from the first order ones. All first order operators $y^{\prime}+a_{0}(t) y$ are disconjugate.) The procedure for going from $n-1$ to $n$ involves a parameter function.

For the remainder of this paper $I$ denotes any compact or open interval, $C(I)$, the set of real valued continuous functions on $I$ and $C^{\prime}(I)$ the set of real valued functions on $I$ which have continuous first derivatives.

Theorem 1. Given $a_{0}$ in $C(I)$ there exist $a_{1}, \cdots, a_{n-1}$ in $C(I)$ such that (1) is disconjugate. Moreover (1) is disconjugate if and only if there exists $r$ in $C(I)$ and $b_{0}, \cdots, b_{n-2}$ in $C^{\prime}(I)$ such that: 34A01.

AMS (MOS) subject classifications (1970). Primary 34A30, 34A05; Secondary

Key words and phrases. Ordinary differential equations, disconjugacy, factorization of differential operators, Markov systems, Tchebycheff systems, algorithm, constructive characterization.

1 Partially supported by NSF grant GP-44012.

Copyright $\odot$ 1975, American Mathematical Society 


$$
\begin{gathered}
y^{(n-1)}+b_{n-2} y^{(n-2)}+\cdots+b_{0} y \text { is disconjugate and } \\
b_{0}^{\prime}+r b_{0}=a_{0}, \quad b_{i}^{\prime}+r b_{i}+b_{i-1}=a_{i} \text { for } i=1, \cdots, n-2,
\end{gathered}
$$

$$
b_{n-2}+r=a_{n-1} \text {. }
$$

From Theorem 1 the following algorithm for the construction of $n$th order disconjugate operators of type (1) is obtained: Start with $a_{0}$ in $C(I)$. Choose any $r$ in $C(I)$. Let $b_{0}$ be any solution of $x^{\prime}+r x=a_{0}$ i.e.,

$$
b_{0}(t)=\exp \left(-\int_{t_{0}}^{t} r(s) d s\right)\left[c+\int_{t_{0}}^{t} \exp \left(\int_{t_{0}}^{u} r(s) d s\right) a_{0}(u) d u\right]
$$

for any $t_{0}$ in $I$ and constant $c$. Choose $b_{1}, \cdots, b_{n-2}$ such that (i) holds. Determine $a_{i}$ for $i=1, \cdots, n-1$ by the second and third equations under (ii). Then the operator $L$ determined by (1) is disconjugate. Furthermore all disconjugate operators $L$ of type (1) on open or compact intervals are obtained this way.

For purposes of illustration we discuss this construction for the cases $n=2$ and $n=3$. The case $n=2: L y=y^{\prime \prime}+a_{1} y^{\prime}+a_{0} y$. The idea is to start with a given $a_{0}$ in $C(I)$ and characterize all functions $a_{1}$ for which $L$ is disconjugate. The characterization is

$$
a_{1}(t)=r(t)+\exp \left(-\int_{t_{0}}^{t} r(s) d s\right)\left[c+\int_{t_{0}}^{t} \exp \left(\int_{t_{0}}^{u} r(s) d s\right) a_{0}(u) d u\right]
$$

where $t_{0}$ is any point in $I, c$ is an arbitrary constant, and $r$ is an arbitrary function in $C(I)$.

In the literature, disconjugacy of second order equations is usually discussed for equations in the form $y^{\prime \prime}+q y$ or $\left(r y^{\prime}\right)^{\prime}+q y$. Consider $y^{\prime \prime}+$ qy. The question of disconjugacy for this equation reduces to: When is $a_{1}$ in (3) (with $a_{0}=q$ ) zero? Setting $a_{1}(t) \equiv 0$ and differentiating reduces (3) to $r^{\prime}+r^{2}+a_{0} \equiv 0$. This is the famous Riccati equation associated with the form $y^{\prime \prime}+a_{0} y$. So our characterization (3)-in the second order case for the special form $y^{\prime \prime}+a_{0} y$-reduces to the well-known equivalence between disconjugacy and existence of solutions of the Riccati equation.

The case $n=3: L y=y^{\prime \prime \prime}+a_{2} y^{\prime \prime}+a_{1} y^{\prime}+a_{0} y$. Again the idea is to start with any function $a_{0}$ and determine all pairs of functions $\left(a_{1}, a_{2}\right)$ which make $L$ disconjugate. This is done as follows: Let $r$ be in $C(I)$ and let $b_{0}$ be any solution of $x^{\prime}+r x=a_{0}$. Take any $b_{1}$ for which 
$y^{\prime \prime}+b_{1} y+b_{0} y$ is disconjugate (i.e., determine $b_{1}$ as discussed in the case $n=2$ above). Now let $a_{1}=b_{1}^{\prime}+b_{0}+r b_{1}$ and $a_{2}=r+b_{1}$. Then $L$ is disconjugate and all third order disconjugate operators $L$ of type (1) on open or compact intervals are obtained this way.

The proof of Theorem 1 is based on the following idea: The $n$th order operators (1) which are disconjugate are precisely those determined by products

$$
\left(y^{\prime}+r y\right)\left(y^{(n-1)}+b_{n-2} y^{(n-2)}+\cdots+b_{0} y\right)
$$

where the $(n-1)$ st order operator with coefficients $b_{i}$ is disconjugate. Product here is meant in the sense of composition. A detailed proof and related matters will be published elsewhere.

DEPARTMENT OF MATHEMATICAL SCIENCES, NORTHERN ILLINOIS UNIVERSITY, DEKALB, ILLINOIS 60115

Current address: Department of Mathematics, The University, Dundee, Scotland 